\title{
Mitochondrial and oxidative stress genes are differentially expressed in neutrophils of sJIA patients treated with tocilizumab: a pilot microarray study
}

Ebun Omoyinmi ${ }^{1 *}$, Raja Hamaoui ${ }^{2}$, Annette Bryant ${ }^{2}$, Mike Chao Jiang ${ }^{1}$, Trin Athigapanich ${ }^{1}$, Despina Eleftheriou ${ }^{1}$, Mike Hubank ${ }^{3}$, Paul Brogan ${ }^{1}$ and Patricia Woo ${ }^{2}$

\begin{abstract}
Background: Various pathways involved in the pathogenesis of sJIA have been identified through gene expression profiling in peripheral blood mononuclear cells (PBMC), but not in neutrophils. Since neutrophils are important in tissue damage during inflammation, and are elevated as part of the acute phase response, we hypothesised that neutrophil pathways could also be important in the pathogenesis of SJIA. We therefore studied the gene profile in both PBMC and neutrophils of sJIA patients treated with tocilizumab.

Methods: We studied the transcriptomes of peripheral blood mononuclear cells (PBMC) and neutrophils from eight paired samples obtained from $4 \mathrm{sJlA}$ patients taken before and after treatment, selected on the basis that they achieved ACR90 responses within 12 weeks of therapy initiation with tocilizumab. RNA was extracted and gene expression profiling was performed using Affymetrix GeneChip Human Genome U133 Plus 2.0 microarray platform. A longitudinal analysis using paired t-test $(p<0.05$ and $F C \geq 1.5)$ was applied to identify differentially expressed genes (DEGs) between the two time points followed by ingenuity pathway analysis. Gene Set Enrichment Analysis (GSEA) and quantitative real-time PCR were then performed to verify the microarray results.

Results: Gene ontology analysis in neutrophils revealed that response to tocilizumab significantly altered genes regulating mitochondrial dysfunction and oxidative stress $(p=4.6 \mathrm{E}-05)$. This was independently verified with GSEA, by identifying a set of oxidative genes whose expression correlated with response to tocilizumab. In PBMC, treatment of sJIA with tocilizumab appeared to affect genes in Oncostatin M signalling and B cell pathways.
\end{abstract}

Conclusions: For the first time we demonstrate that neutrophils from sJIA patients responding to tocilizumab showed significantly different changes in gene expression. These data could highlight the importance of mitochondrial genes that modulate oxidative stress in the pathogenesis of $\mathrm{s} J \mathrm{~A}$.

Keywords: Systemic juvenile idiopathic arthritis (sJIA), Anakinra, Tocilizumab, Gene expression, Neutrophils

\footnotetext{
*Correspondence: e.omoyinmi@ucl.ac.uk

${ }^{1}$ Infection, Inflammation and Rheumatology Section, Institute of Child Health,

UCL, London, UK

Full list of author information is available at the end of the article
} 


\section{Background}

Juvenile Idiopathic Arthritis (JIA) is a heterogeneous group of arthritides classified into 7 subtypes by the International League of Associations for Rheumatology (ILAR) [1]. We have previously examined the transcriptome of patients with systemic JIA (sJIA), a distinct clinical subgroup with characteristic systemic inflammation as well as arthritis [2]. Systemic inflammatory features of this group of patients include quotidian fever, evanescent skin rash, lymphadenopathy, serositis and hepatosplenomegaly. These clinical features are reminiscent of the heritable autoinflammatory diseases; consequently, sJIA is frequently referred to as an autoinflammatory disease because of these clinical manifestations, lack of autoantibodies, and association with variants in inflammatory and anti-inflammatory cytokine genes, including IL-1, IL-6, and IL-10 [3-8]. High or abnormal production of inflammatory cytokines in the plasma, and impressive results from clinical trials to modify IL- 1 and IL- 6 cell signalling have provided further evidence for the important roles of these cytokines in the pathogenesis of sJIA [9-17].

The molecular pathways leading to the symptoms of sJIA are not yet fully understood, but have been clarified to some extent during recent years by the use of microarray technology. So far, gene expression studies have been performed mostly on peripheral blood mononuclear cells (PBMC) from sJIA patients, with the exception of one study which examined whole blood [18]. Studies of this nature have led to the identification of gene clusters associated with disease activity [2] and severity [19], and revealed dysregulation of cytokine pathways [9, 12, 18]. A study applied DNA microarray methods across different subtypes of JIA, and identified patterns of gene expression that correlated with clinical characteristics of the different subtypes. In particular, it was shown that sJIA is distinct from Oligoarticular and Polyarticular JIA [20]. Furthermore, gene expression profiles in neutrophils from children with polyarticular JIA demonstrate defects in genes modulated by IFN-gamma and IL-8 [21-23].

Since many autoinflammatory diseases involve the genes and pathways of the innate immune system, we proposed that neutrophils were worthy of further study in sJIA. Neutrophils are highly specialised leucocytes that mediate inflammatory changes in tissues, and are important effectors of innate/early immune responses [24]. Once activated, neutrophils can produce reactive oxygen species that neutralise the effects of bacteria and fungi, and together with chemokines and other inflammatory mediators, orchestrate inflammation in tissues [24]. In autoinflammatory diseases, abundant neutrophils are usually seen in sites of inflammation, often in the relative absence of lymphocytes $[25,26]$. Consequently, we hypothesised that neutrophils are important in the pathogenesis of sJIA, and that there would be important differences in the differentially expressed genes in response to tocilizumab.

\section{Methods \\ Patients and treatment}

This pilot study included symptomatic sJIA patients with disease duration ranging from 9 months to 7 years (Table 1), who were selected for analysis on the basis that they achieved an adapted American College of Rheumatology response of 90 (ACR90) to tocilizumab. The clinical features of the patients in this study are shown in Table 1. All patients provided written informed consent to enter this observational study, with ethical approval by Great Ormond Street Hospital for Children NHS Trust (GOSH)/Institute of Child Health Research Ethics committee (registration number 02RU06). We examined gene expression profiles before, and 3 months after treatment with tocilizumab (a humanised monoclonal antibody targeting the interleukin 6 receptor, received as routine clinical care) at GOSH. The patients received tocilizumab using a standard recommended dosing regimen [14, 16]; samples from responders were collected from November 2008 to July 2009. All patients fulfilled sJIA ILAR classification criteria [27]. Patients were considered for this study group if they had persistent signs of inflammation refractory to therapy with methotrexate (MTX), with or without corticosteroid or anti-TNF- $\alpha$ therapy. Blood samples referred to as "Before" were obtained from all patients at routine baseline screening prior to the initiation of treatment when the disease status was active. Twelve weeks from enrolment into the study, an "After" sample was collected. Clinical assessment of disease activity was determined using the JIA core set criteria and definition of improvement [28]. The American College of Rheumatology (ACR) paediatric adapted improvement score was then calculated at the time of sample collection (28). Response was defined as: paediatricadapted American College of Rheumatology (ACR) 90 response, plus normalisation of the $C$ reactive protein (CRP) and erythrocyte sedimentation rate (ESR).

\section{Leucocyte separation}

PBMC were obtained from Ficoll separation of whole blood using Lymphoprep reagent (Stemcell Technologies). Neutrophils were subsequently isolated from the granulocyte and red cell pellet formed at the bottom of the Ficoll tube by hypotonic cell lysis in ammonium chloride buffer. Cell viability was assessed by trypan blue dye exclusion. Purified cells were visualised and counted using a haemocytometer before re-suspension in TRIzol reagent (Invitrogen, Paisley, UK). Care was taken to minimize the time between blood-drawing and placing cells in TRIzol reagent to within $3 \mathrm{~h}$. 
Table 1 Clinical features of patients and response to tocilizumab

\begin{tabular}{|c|c|c|c|c|c|c|c|c|}
\hline Sample code & $37-5$ & $37-7$ & $50-2$ & $50-4$ & $51-1$ & $51-3$ & $53-2$ & $53-4$ \\
\hline Sample type & $\mathrm{N}$ & N & $P, N$ & $P, N$ & $P, N$ & $P, N$ & $P, N$ & $P, N$ \\
\hline Conditions & Pre-TOC & 12 weeks TOC & Pre-TOC & 12 weeks TOC & Pre-TOC & 12 weeks TOC & Pre-TOC & 12 weeks TOC \\
\hline Ethnicity & Caucasian & & Caucasian & & Asian & & Caucasian & \\
\hline Gender & Male & & Female & & Male & & Female & \\
\hline Age at onset (AAO) & 7 years & & 9 years & & 14 years & & 4 years & \\
\hline Disease duration & 3.4 years & & 7 years & & 10 month & & 9 months & \\
\hline Fever & Yes & No & No & No & No & No & Yes & No \\
\hline Rash & Yes & No & No & No & No & No & No & No \\
\hline Total WCC (lymphocytes) $\times 10^{9} / \mathrm{L}$ & $22.22(1.36)$ & $10.76(4.99)$ & $10.51(1.28)$ & $5.58(2.67)$ & N/D & $5.84(2.52)$ & $13.98(4.80)$ & $8.09(4.03)$ \\
\hline CRP (mg/l), 0-20 normal range & 84 & $<5$ & 24 & $<5$ & 13.6 & $<3$ & 56 & $<5$ \\
\hline ESR (mm/h), 0-10 normal range & 65 & $<1$ & 29 & 3 & N/D & 1 & 35 & $<1$ \\
\hline Joint active & 6 & 0 & 22 & 10 & 6 & 0 & 51 & 0 \\
\hline Joint limited motion & 6 & 0 & 30 & 12 & 7 & 0 & 49 & 3 \\
\hline Parental VAS & N/D & 0 & 6.8 & 1 & 2.8 & 0.4 & 5 & 2.2 \\
\hline Physician VAS & 5 & 0 & 8 & 4 & 3 & 1 & 8 & 0.4 \\
\hline CHAQ & 1.75 & 0 & 1.5 & 0.38 & 0 & 0 & 1.13 & 0.5 \\
\hline ACR & - & 90 & - & 90 & - & 90 & - & 90 \\
\hline
\end{tabular}

The demographics, clinical and laboratory parameters of the patients whose peripheral blood was used in this study are shown. For each patient, baseline measurements were taken before starting tocilizumab (TOC) treatment, and 12 weeks later. Normal range for total white cell count (WCC) is $4.5-13.5 \times 10^{9} / \mathrm{L}$ and for lymphocytes $1.5-7 \times 10^{9} / \mathrm{L}$. The different types of samples taken/analysed were: PBMC $=\mathrm{P}$; and Neutrophils $=\mathrm{N}$. There are 3 paired samples for PBMC $(\mathrm{P})$ and 4 for neutrophils (N). Response to treatment was determined by ACR90 response definition of improvement in juvenile arthritis [47], plus normalisation of the erythrocyte sedimentation rate (ESR) and $C$ reactive protein (CRP). N/D not determined, VAS visual analogue score, CHAQ child health assessment questionnaire, $A C R$ American College of Rheumatology

\section{Assessment of purity and the effect of isolation process on neutrophil activation status}

We used two-colour flow cytometric analysis to monitor ex vivo activation of neutrophils as a result of sample manipulation. Antibody staining for surface markers was performed as previously described [29], using PE conjugated mouse Anti-Human CD11b/Mac-1 (a marker to monitor ex vivo activation of neutrophils), and APC-Cy7 mouse Anti-Human CD16, expressed on the surface of neutrophils. A FACScan flow cytometer and CellQuest analysis software were used for the acquisition and analysis of the data. The purity and activation status of isolated neutrophils were evaluated by gating on the CD11b/Mac-1 and CD16 double-positive cells.

\section{Lipopolysaccharide-induced activation of isolated neutrophils}

Isolated neutrophils at a density of $2 \times 10^{6}$ cells $/ \mathrm{ml}$ in $\mathrm{RPMI}$ were cultured at $37^{\circ} \mathrm{C}$ for $1 \mathrm{~h}$ in the presence or absence of $1 \mu \mathrm{g} / \mathrm{ml}$ lipopolysaccharide (LPS). The level of neutrophil activation was again evaluated by flow cytometry analysis of CD11b/Mac-1 on CD16 positive cells.

\section{Microarray procedures}

The protocols for RNA extraction and microarray hybridization to Affymetrix U133 plus 2.0 arrays (Affymetrix, Santa Clara, CA), which includes approximately 54,000 probe sets representing 47,400 human transcripts were as previously described [2]. Processing of Affymetrix data was performed in GeneSpring GX11.0 (Agilent) using GCRMA (RMA; Robust Multi-array Analysis that accounts for the probes GC content) method for normalizing and summarizing probe-level intensity measurements [30]. Probe sets with very low absolute expression intensity values $(<10)$ in all patients in either the "before" or "after" samples were filtered out since at this level it would be difficult to distinguish a true effect from background noise [31]. Multiple probe sets mapping to the same gene that passed this filter were retained and the differential expression of any probe set for a given gene was used as a surrogate for differential gene expression. The affymetrix data files have been submitted to NCBI Gene Expression Omnibus (GEO; http://www.ncbi.nlm.nih.gov/ geo/) under accession number GSE76492.

\section{Statistical analysis of microarray data}

Paired t-test of the "before" and "after" treatment samples at statistical threshold of $p<0.05$ with fold-change (FC) 
$\geq 1.5$ was considered as significant for identification of differentially expressed genes. Both unsupervised and supervised hierarchical clustering was performed using Pearson's centered correlation and Ward's linkage rule within GeneSpring software to generate heatmaps of gene expression profiles.

\section{Ingenuity Pathway Analysis (IPA)}

The differentially expressed probe sets identified with statistical testing were uploaded into the IPA knowledge database (version 7.2, Ingenuity Systems, www.ingenuity.com) to explore the pathways that were significantly associated with each dataset. In the case of multiple probe sets mapping to the same transcript, IPA considered the probe set with the highest fold change for the pathway analysis. Each annotated gene was mapped to its corresponding gene object in the IPA Knowledge Base. The Affymetrix U133 plus 2.0 array was selected as background or reference dataset for calculation of significant functions/pathways. The core analysis was run using the following setting in IPA: data source included all species, tissues and cell lines; interactions were queried on all genes stored within IPA; and no fold-change cut-off was specified. The significance of the association between the data set and the canonical pathways was calculated in 2 ways according to IPA user's manual: 1) a ratio of the number of genes from our dataset that map to the pathway, divided by the total number of genes that make up the pathway; 2) Fisher's exact test was used to calculate a $p$-value determining the probability that the association between the genes in the data set and the canonical pathway was explained by chance alone. A pathway was considered significant with a $p$-value that was less than 0.05 . All pathways were named according to the terminology used in IPA.

\section{Gene Set enrichment analysis of microarray data}

GSEA was performed using gene sets from MSigDB v2.5 gene set database (http://www.broadinstitute.org/gsea/ msigdb/). This analytical technique is designed to test a priori defined gene sets (for example, pathways) for association with phenotypes [32]. In brief, the method consists of the following steps: list of genes are first ranked by Signal2Noise metric using the correlation between their expression and the class distinction (for example, "before" versus "after" treatment samples). Given a defined set of genes (for example, genes members of a signalling pathway), the goal of GSEA is to determine whether the members of the gene set are found at the top or bottom of the list, indicating that they are associate with the phenotypic distinction, rather than being distributed uniformly or randomly across the list. An enrichment score is then calculated to quantify the degree to which a gene set is over-represented at the top or bottom of the entire ranked list. After calculation of the enrichment scores, statistical significance test was done with 1,000 permutation of genes using the weighted to enrichment statistic.

\section{Quantitative real-time (qRT-PCR)}

cDNA was generated from 500ng of total RNA using High Capacity RNA-to-cDNA kit (Life Technologies) according to the manufacturer's instruction. qRT-PCR assays were performed using QuantiTect SYBR Green PCR kit and the following commercially available QuantiTect primers: (Qiagen, Crawley, UK) for COX6C (Assay ID QT00221137), NDUFB2 (Assay ID QT00050904), and LAT (Assay ID QT00232127). Differences in expression were determined by the relative quantification method; the cycle threshold $\left(C_{T}\right)$ values of the target genes were first normalised to the $C_{\mathrm{T}}$ values of endogenous control large ribosomal protein P0 (RPLPO, Assay ID QT00075012).

\section{Results}

Patients

This exploratory analysis included only sJIA patients with active symptomatic disease who were selected on the basis that they responded (by attaining ACR90) to tocilizumab (Table 1). All the patients described herein additionally had normal CRP/ESR at second sample collection with an ACR90 response, and therefore all were considered as responders for the purposes of this study.

\section{Identification of genes/pathways associated with good response to tocilizumab}

After filtering out unreliable probe sets with expression at background level, 23,895 and 20,408 out of 54,459 probe sets were considered as expressed in PBMC and neutrophils respectively. Unsupervised hierarchical clustering $(\mathrm{HC})$ of all expressed genes showed that the pre- and post-treatment samples from the patients ( 3 sIIA patients for PBMC samples and 4 sJIA patients for neutrophils) clustered together for each cell type (data not shown). Therefore, paired t-test of gene expression in each individual before and after treatment is preferred for the analysis of change over time (with statistical cutoff level of $p<0.05$ with $\geq \mathrm{FC} \mathrm{1.5)} \mathrm{to} \mathrm{cross} \mathrm{sectional}$ analysis of the time points. The full lists of the genes are given in Additional files 1 (for PBMC) and 2 (for neutrophils). We performed supervised $\mathrm{HC}$ of the differentially expressed probe sets in PBMC and neutrophils separately, and generated heatmaps to illustrate the expression pattern between the two time points (Fig. 1a-b). This analysis yielded two main clusters of probe sets for each of the 2 cell types; cluster 1 for probe sets with decreased expression at 12 weeks, and cluster 2 corresponding to probe sets with increased expression at 12 weeks. 

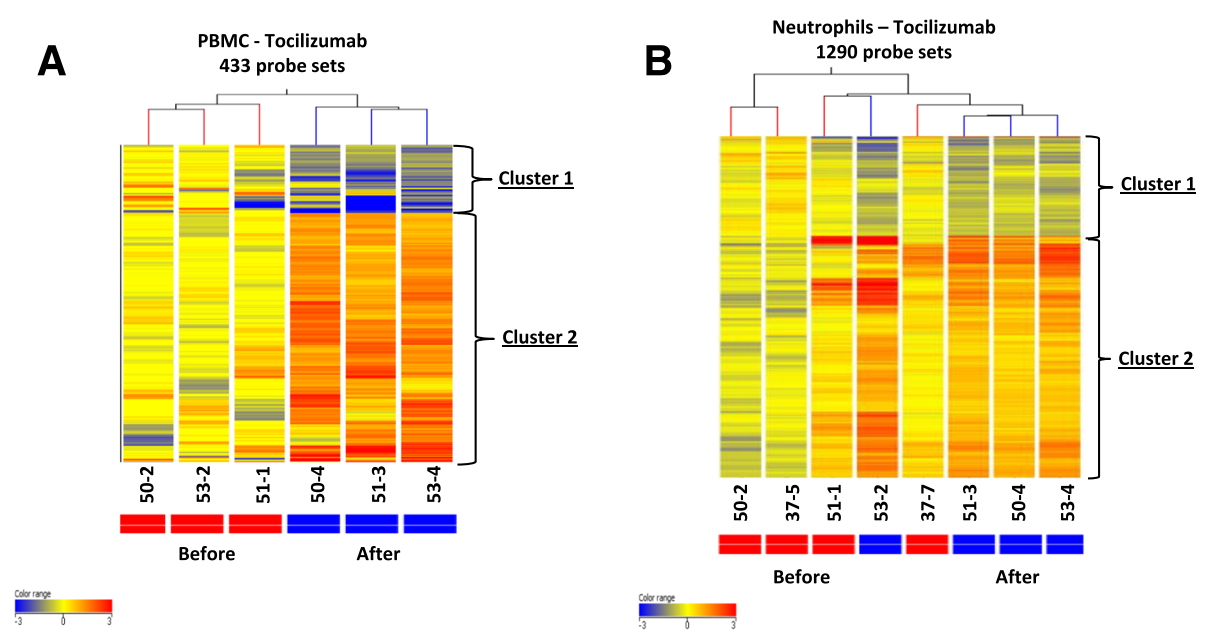

Fig. 1 Heatmaps illustrating supervised hierarchical clustering analysis of the probe sets differentially expressed in PBMC (a) and Neutrophils (b) from sJIA patients before and after tocilizumab treatment ( $3 \mathrm{sJIA}$ patients for PBMC samples and $4 \mathrm{sJIA}$ patients for neutrophils). The differential expression of any probe set for a given gene was used as a surrogate for differential gene expression. Samples were collected at time points zero, and 3 months post treatment. All were responders with ACR90 (Table 1). Clustering analysis was performed in GeneSpring (GX11) as described in Methods. Normalized expression is colour coded in which red is high and blue is low relative to the median of the 'before' treatment samples. In the heatmaps, each column represents a sample and each row represents a gene. Full list of all genes in clusters are available in Additional files 1 and 2. However, listed below are genes with fold change $(F C) \geq 3$ found within the clusters in the order of decreasing $F C$ values. A cluster 1 : Genes with significant decrease (FC $\geq 3$ fold) after treatment with tocilizumab in PBMC: FCGR3B, KCNJ15, CHI3L1, ADM, PROS1, SOCS3, CHI3L1, and NRG1. A cluster 2: there were genes with increased expression on the heatmap but these were all less than 3 fold change. B cluster 1: Genes with significant decrease ( $F C \geq 3$ fold) after treatment with tocilizumab in neutrophils: ARHGAP24, CLEC5A, TAF8. B cluster 2: Genes with significant increase (FC $\geq 3$ fold) after treatment with tocilizumab in neutrophils: CD3D, LOC129293, AQP3, LAT, LY9, HLA-DPB1, TRA@, GFI1B, BCL11B, PASK, POLR3E, DOK2, AFG3L2, MEX3C, PASK, ENTPD6, KIAA0114, FAM102A, RCAN3, ATXN10, TNFAIP8L1, ABHD14B, RPL10A, GPR44, ATP6VOE2, ADARB1, APEX1, C17orf61, KLHL3, MRPS24, POU6F1, LDLRAP1, NDUFB2, SLC25A38, UBQLN4, KLF10, C220rf32, AKR1B1, PPP3CC, GSS, CAMK1, EIF3C, EEF2K, ILF3, RPL13, SLC25A6, THEM4, RPL13A, RDH14, KCTD15, DNMT1, TTC4, KIAA0748, AKR7A2, PLSCR3, ZNF639, KIAA1024, UNC84A, IARS, C110rf31, PVT1, DNPEP, LOC202781, LAGE3, NHP2, LSG1, SIRPG, SLC35B2, EEF2K, AES, TMEM14A, PAN2, DDX39, NOC4L, CAMSAP1, LOC100131731, BHLHE40, ECHS1, CLNS1A, CPSF1, LOC93622, TOMM5, COX6C, NLRC3, EIF3B, CIRH1A, OLIG1, ZBTB40

We used Ingenuity Pathway Analysis (IPA) tools to identify the significant pathways $(p<0.05)$ associated with the 2 lists of differentially expressed probe sets in PBMC and neutrophils. A summary of the results are presented in Table 2 for the top 10 IPA canonical pathways presented in order of significance.

\section{PBMC gene expression profiling in tocilizumab treated patients showed changes in pathways associated with IL-6 signalling}

Only 18 genes out of 385 differentially expressed unique genes in PBMC of sJIA patients were significantly mapped to 6 IPA canonical pathways (Table 2, Additional file 3). The most significant pathway was the Oncostatin M signalling pathway from this analysis; $p=0.015$, with 3 mapped genes (IL6ST, KRAS, CHI3L1).

\section{Neutrophil gene expression profiling demonstrated changes in mitochondrial and $T$ cell pathways}

The most significant change in IPA pathway for neutrophils was the "mitochondrial dysfunction pathway" $(p=0.000046)$ with increased expression of 20 genes in response to tocilizumab treatment (Table 2, Additional file 4).
Table 2 Top ten IPA pathways that were found to be significantly altered in PBMC and neutrophil samples from sJIA patients responding to tocilizumab

\begin{tabular}{|c|c|}
\hline $\mathrm{PBMC}^{\mathrm{a}}$ & Neutrophils \\
\hline Oncostatin M signalling (3) & Mitochondrial dysfunction (20) \\
\hline Natural killer cell signalling (5) & EIF2 signalling (23) \\
\hline Glutamine biosynthesis I (1) & $\begin{array}{l}\text { NRF2-mediated oxidative stress } \\
\text { response (20) }\end{array}$ \\
\hline B Cell receptor signalling (6) & $\begin{array}{l}\text { Calcium-induced T lymphocyte } \\
\text { apoptosis (9) }\end{array}$ \\
\hline PPARa/RXRa activation (6) & mTOR signalling (20) \\
\hline \multirow[t]{5}{*}{ Thyroid hormone biosynthesis (1) } & Sucrose degradation V (Mammalian) (3) \\
\hline & $\begin{array}{l}\text { Regulation of elF4 and p70S6K } \\
\text { signalling (16) }\end{array}$ \\
\hline & TCA cycle II (Eukaryotic) (5) \\
\hline & $\begin{array}{l}\text { CTLA4 signalling in cytotoxic T } \\
\text { lymphocytes (11) }\end{array}$ \\
\hline & Protein ubiquitination pathway (24) \\
\hline
\end{tabular}

There were only 6 significant canonical pathways for this condition. In brackets are the numbers of genes from the input file in each pathway 
These genes included ATP5A1, an ATP synthase of complex $\mathrm{V}$, and 13 other genes that code for subunits of other mitochondria respiratory chain complexes. In complex I there was increased expression of 8 nuclear encoded NADH dehydrogenase genes of which NDUFB2 had the highest $\mathrm{FC}$ value of 3 . Ubiquinol-cytochrome $\mathrm{c}$ reductase (UQCRH) coding for a hinge protein was the only gene regulated in complex III; in complex IV there was increased expression of nuclear encoded cytochrome c oxidase; Cox $5 A$, Cox6C and Cox7C. The expression of linker for activated cells $(L A T)$ was significantly increased 6-fold in response to tocilizumab treatment, and this gene was also mapped to two significant T cell pathways; CD28 Signalling in T Helper Cells and Regulation of IL-2 Expression in Activated and Anergic T Lymphocytes.

\section{GSEA shows enrichment of mitochondrial/oxidative phosphorylation genes in SJA patients treated with tocilizumab}

We analysed the microarray data by GSEA [32] to determine whether the differential expression of mitochondrial genes observed in neutrophils of sJIA patients treated with tocilizumab were truly representative of the global gene expression data without regard to the accuracy of statistical threshold applied in the initial analysis. For GSEA, we extracted from MSigDB v2.5 gene set database (http://www.broadinstitute.org/gsea/msigdb/) the KEGGS_oxidative phosphorylation gene set which originally contained 135 genes, but the analysis was restricted to 110 genes present on the Affymetrix U133 plus 2.0 chip.
This gene set was chosen because it is the best representative of the IPA mitochondrial dysfunction pathway as it contains 12 of the 20 genes mapped to this pathway (indicated with an asterisk in the Additional file 4). The result of GSEA showed significant enrichment of the oxidative phosphorylation gene set (Additional file 5 for the gene list) with increased expression of the leading edge gene subset in sJIA patients post tocilizumab treatment (Fig. 2a, b).

\section{Verification of Microarray analysis by qRT-PCR}

Given the limitation in the blood sample volume, it was only possible to verify the accuracy of our microarray analysis by real-time quantitative PCR on just the neutrophil samples used in the array experiment. The qRT-

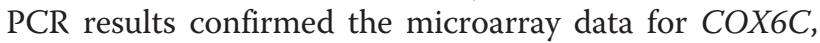
$L A T$ and NDUFB2 (Fig. 2c).

\section{Minor change in neutrophil activation following sample manipulation}

Before the microarray analysis of gene expression in neutrophils, we carried out a series of control experiments where we compared expression level of the neutrophil activation marker CD11b/Mac-1 measured in geometric mean fluorescence intensity (GMFI) on CD16+ cells gated on granulocytes population within whole blood to that of isolated neutrophils $\pm 1 \mu \mathrm{g} / \mathrm{ml}$ LPS by flow cytometry. These experiments were performed in healthy controls as well as sJIA patients with active and inactive disease. There was a small increase in $\mathrm{CD} 11 \mathrm{~b}$ expression due to

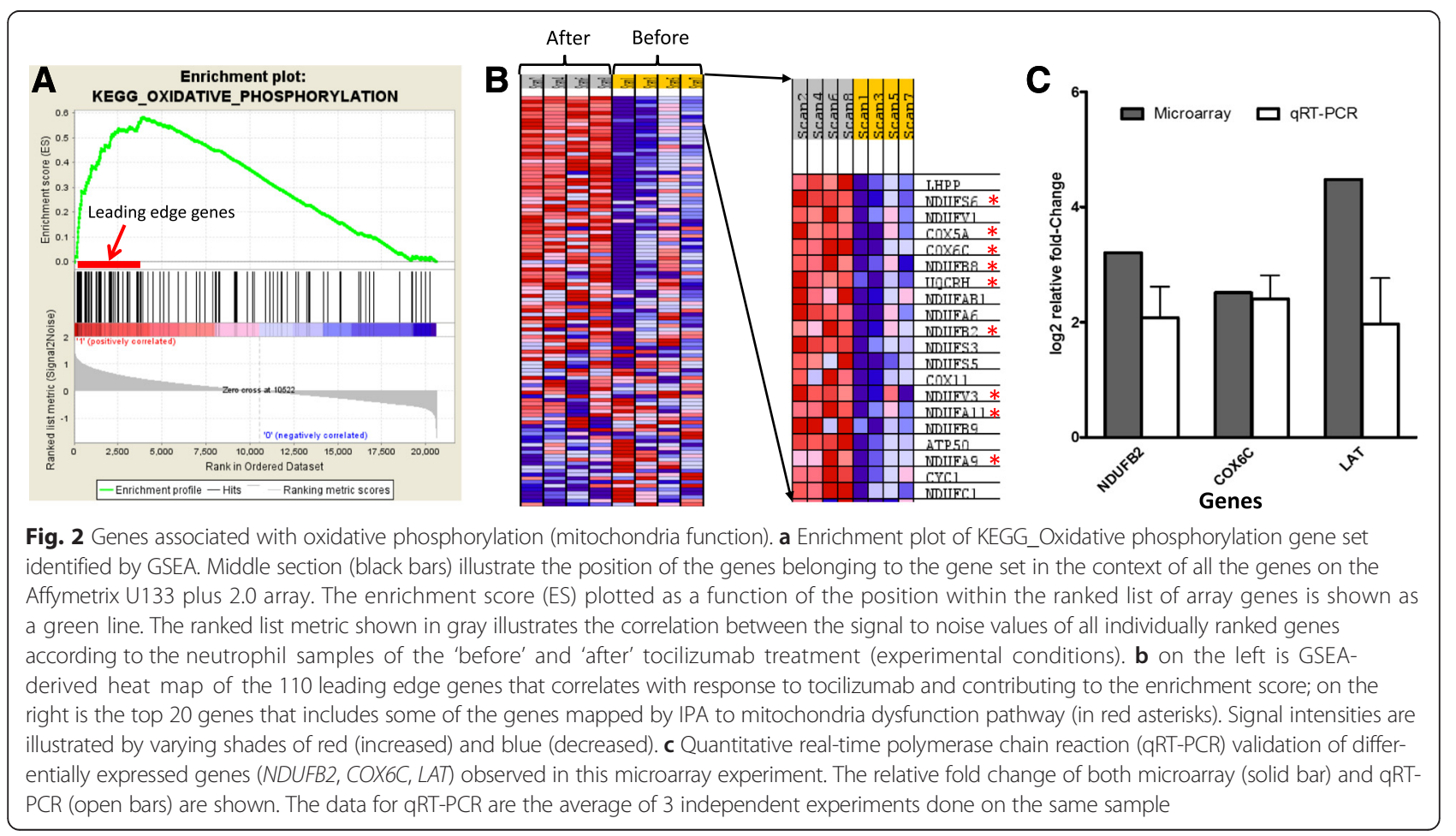


the separation process. However, neutrophils were still relatively inactive since they were highly sensitive $(>10$ fold increase) to further activation when stimulated with LPS in both healthy controls and sJIA patients (Additional file 6). The purity of isolated neutrophils as defined from the double positive cells was >96\% (Additional file 7). The median \pm standard deviation (SD) of double positive isolated neutrophils for all the sJIA samples was $96.55 \% \pm 0.63$ compared to $60 \% \pm$ 6.89 in whole blood (Additional file 7).

\section{Discussion}

A novel and important aspect of this study is that we examined neutrophils in addition to PBMC since these cells are known to play important roles in innate immune responses and the pathogenesis of sJIA [33-35], but previously have not been studied by transcriptome analyses in this context. Both IPA and GSEA showed significant correlation between mitochondrial/oxidative stress genes and response to tocilizumab in neutrophils of sJIA patients. Our microarray data indicated a significant increase in the expression levels of nuclear encoded mitochondrial genes that are involved in electron transport (e.g. NDUFB2, COX6C, both confirmed by q RT-PCR) after a good clinical response to tocilizumab; or conversely, that decreased gene expression was present in those with active disease. This observation is consistent with the study by Ishikawa et al. using whole blood for gene profiling, showing reduced expression of mitochondrial DNA-encoded genes in SIIA patients with active disease compared to healthy controls [18]. They did not detect differences in any of the nuclear encoded genes including those found in this study, which might be due to differences in study design, analysis of a mixed cell population of whole blood (PAXgene) samples, microarray platforms and/or analytical methods used. Our data are further supported by the fact that IL- 6 has been shown to have a direct effect on mitochondrial function by decreasing both the membrane potential and ATP production with subsequent increase in intracellular reactive oxygen species (ROS) level in an in vitro study [36]. Currently, there is growing evidence that mitochondrial ROS and defective antioxidant responses play an important role in the pathogenesis of a number of autoinflammatory and autoimmune diseases [37-42]. Given that IL-6 is one of the most important mediators of fever and the acute phase response (including neutrophilia), and that neutrophil mitochondrial ROS are increasingly understood to be important in autoinflammation, it is perhaps unsurprising that we have detected perturbation of mitochondrial genes in neutrophils in active sJIA, which change in response to successful therapy with IL-6 blockade. This further emphasises the potential importance of studying neutrophils in sJIA and its response to treatment in the future.
It was striking and unexpected to observe the differential expression of $\mathrm{T}$-cell receptor associated genes such as delta 3 molecule $(C D 3 D)$, zeta-chain tyrosine kinase (Zap70) and linker for activation of $\mathrm{T}$ cell (LAT, confirmed by q RT-PCR) in the neutrophil cell population from patients treated with tocilizumab. Although we cannot rule out minor and uneven contamination of the separated cell populations by $\mathrm{T}$ cells, previous studies have revealed that human neutrophils do express TCR including components of the signalling complex [43, 44], but their exact role in neutrophils is not yet known. Our microarray data showed that $L A T$ is expressed in neutrophils of all the 4 patients studied. Furthermore Puellmann et al. addressed the issue of contamination by clearly demonstrating that the neutrophil cell populations were negative for $\mathrm{T}$ cell markers (CD3, CD4, and CD8) [44]. These findings suggest that neutrophils may have the potential to mediate both innate and adaptive immune responses in sJIA patients.

It was reassuring to note that in the DEGs from the PBMC of tocilizumab-treated sJIA patients, there was decreased expression of SOCS3, a suppressor of cytokine signalling, consistent with the fact that tocilizumab decreases intracellular IL-6 signalling and therefore reduction in the expression of SOCS3. We have previously reported that specific innate immune genes are upregulated in PBMC of sJIA patients during active disease [2]. In the same study we also demonstrated that $B$ cells express higher levels of IL- 6 than monocytes. Given the heterogeneous composition of PBMC samples, it is possible that the significant $\mathrm{B}$ cell pathway and oncostatin $M$ signalling observed in this study might be from $B$ cells, further illustrating the importance of examining a homogeneous population of cells.

One obvious limitation of this study is that the sample sizes are small, and therefore deemed likely to generate a number of false positive genes. This effect is alleviated to some extent by a prospective approach in sample collection and paired sample analyses. The independent application of GSEA provided a strong supporting evidence for the observed increased expression of mitochondrial gene sets following good response to tocilizumab, thereby validating this finding. We have also identified genes/pathways previously known to be involved in the pathogenesis of sJIA, suggesting that our findings are not false positives.

It might be potentially important and interesting to profile patients who do not clinically respond to IL-6R blockade, assuming that their pathology is via a different pathway. We have not addressed this question in this initial study because the lack of the clinical response to tocilizumab is a continuum that may be due to many reasons. We feel that using a clinically well-defined full response to drug therapy of ACR90 is better to observe 
significant differences and also allows us to collect sufficiently similar patients to do statistical comparisons.

\section{Conclusions}

In conclusion, we have shown that mitochondrial genes in neutrophils could play a role in the pathogenesis of some sJIA patients, and highlight that it is important to examine the neutrophils in future transcriptome analyses in SJIA, as has already been shown in polyarticular JIA [21-23, 45, 46].

\section{Additional files}

Additional file 1: File contains the list of 433 significant probe sets obtained by paired t-test using the statistical cut-off level of $p<0.05$ with $\mathrm{FC} \geq 1.5$ in PBMC from $\mathrm{SJIA}$ patients successfully treated with tocilizumab. (XLS $86 \mathrm{~kb}$ )

Additional file 2: File contains the list of 1290 significant probe sets obtained by paired t-test using the statistical cut-off level of $p<0.05$ with $\mathrm{FC} \geq 1.5$ in neutrophils from sJIA patients successfully treated with tocilizumab. (XLS $227 \mathrm{~kb}$ )

Additional file 3: File contains the significant IPA canonical pathways obtained in PBMC from sJIA patients successfully treated with tocilizumab. (XLSX $12 \mathrm{~kb}$ )

Additional file 4: File contains the significant IPA canonical pathways obtained in neutrophils from sJIA patients successfully treated with tocilizumab. (XLSX $22 \mathrm{~kb}$ )

Additional file 5: File contains the enriched genes for KEGG_Oxidative Phosphorylation pathway obtained from GSEA. (XLS $13 \mathrm{~kb}$ )

Additional file 6: Changes in Neutrophil CD11b/Mac-1expression following sample manipulation and LPS stimulation. We compared expression level of CD11 b/Mac-1 on the surface of CD16+ cells gated on the granulocyte population within whole blood to those of isolated neutrophils $\pm 1 \mu \mathrm{g} / \mathrm{ml}$ LPS. We examined 4 individual controls, 3 systemic patients with active disease (denoted ' $A$ ') and two with inactive disease (denoted 'l'). (PPTX 65 kb)

Additional file 7: Flow cytometry analysis of neutrophils from sJIA patients. A: representative dot plots of forward and side scatter of unstained whole blood and isolated neutrophils gating on granulocytes. B: evaluation of human cells stained with antihuman CD16-Cy7 (y-axis) and CD11 b/Mac-1-PE (x-axis) conjugated antibodies in whole blood (left panel) and isolated neutrophils (right panel). The percentages of positive cells are indicated in each quadrant. The percentage of double positive cells (B; top right quadrant) is higher in isolated neutrophils (96.5\%) than in whole blood (58.9\%). C: FACS analysis data for isolated neutrophils and whole blood (WB) samples obtained from 4 sJIA patients before and after tocilizumab treatment. Values on the $y$-axis corresponds to percentages of double positive CD11bMac-1+ CD16+ cells. The horizontal bars show median values. (PPTX $49 \mathrm{~kb}$ )

\section{Abbreviations}

ACR: American College of Rheumatology; DEGs: differentially expressed genes; ILAR: International League of Associations for Rheumatology; MTX: methotrexate; PBMC: peripheral blood mononuclear cells; sJIA: Systemic Juvenile Idiopathic Arthritis.

\section{Competing interests}

The authors declare that they have no competing interests.

\section{Authors' contributions}

EO carried out the experiments, data analysis and drafted the manuscript. RH participated in the microarray experiments, acquisition of clinical data and helped to draft the manuscript. AB coordinated the recruitment of patients, acquisition and analysis of clinical data. MCH and TA performed some of the real-time PCR experiments and helped draft the manuscript. DE participated in study design, acquisition of clinical data and helped to draft the manuscript. $\mathrm{MH}$, made substantial contribution to the statistical analysis and interpretation of data and helped to draft the manuscript. PB participated in the design, data interpretation and helped to draft the manuscript. PW conceived the study, participated in its design and coordination, data interpretation, and helped to draft the manuscript. All authors read and approved the final manuscript.

\section{Acknowledgements}

This work was supported by Arthritis Research UK

Written informed consent was obtained from the parents/guardians of participant for publication of their individual details and accompany images in this manuscript. The consent forms are held by the authors and are available on request.

\section{Author details}

${ }^{1}$ Infection, Inflammation and Rheumatology Section, Institute of Child Health, UCL, London, UK. ${ }^{2}$ Centre for Adolescent Rheumatology, University College London, 4th Floor, Rayne Building, 5, University Street, London WC1E 6JF, UK.

${ }^{3}$ UCL Genomics, Institute of Child Health, London, UK.

Received: 23 July 2015 Accepted: 1 February 2016

Published online: 09 February 2016

References

1. Petty RE. Growing pains: the ILAR classification of juvenile idiopathic arthritis. J Rheumatol. 2001;28(5):927-8.

2. Ogilvie EM, Khan A, Hubank M, Kellam P, Woo P. Specific gene expression profiles in systemic juvenile idiopathic arthritis. Arthritis Rheum. 2007;56(6): 1954-65.

3. Fife MS, Ogilvie EM, Kelberman D, Samuel J, Gutierrez A, Humphries SE, et al. Novel IL-6 haplotypes and disease association. Genes Immun. 2005;6(4):367-70.

4. Fife MS, Gutierrez A, Ogilvie EM, Stock CJ, Samuel JM, Thomson W, et al. Novel IL10 gene family associations with systemic juvenile idiopathic arthritis. Arthritis Res Ther. 2006:8(5):R148.

5. Fishman D, Faulds G, Jeffery R, Mohamed-Ali V, Yudkin JS, Humphries S, et al. The effect of novel polymorphisms in the interleukin-6 (IL-6) gene on IL-6 transcription and plasma IL-6 levels, and an association with systemiconset juvenile chronic arthritis. J Clin Invest. 1998:102(7):1369-76.

6. Ogilvie EM, Fife MS, Thompson SD, Twine $\mathrm{N}$, Tsoras M, Moroldo M, et al. The -174G allele of the interleukin- 6 gene confers susceptibility to systemic arthritis in children: a multicenter study using simplex and multiplex juvenile idiopathic arthritis families. Arthritis Rheum. 2003;48(11):3202-6.

7. Omoyinmi E, Forabosco P, Hamaoui R, Bryant A, Hinks A, Ursu S, et al. Association of the IL-10 gene family locus on chromosome 1 with juvenile idiopathic arthritis (JIA). PLoS One. 2012:7(10):e47673.

8. Stock CJ, Ogilvie EM, Samuel JM, Fife M, Lewis CM, Woo P. Comprehensive association study of genetic variants in the IL-1 gene family in systemic juvenile idiopathic arthritis. Genes Immun. 2008;9(4):349-57.

9. Allantaz F, Chaussabel D, Stichweh D, Bennett L, Allman W, Mejias A, et al. Blood leukocyte microarrays to diagnose systemic onset juvenile idiopathic arthritis and follow the response to IL-1 blockade. J Exp Med. 2007;204(9):2131-44.

10. Gattorno M, Piccini A, Lasiglie D, Tassi S, Brisca G, Carta S, et al. The pattern of response to anti-interleukin-1 treatment distinguishes two subsets of patients with systemic-onset juvenile idiopathic arthritis. Arthritis Rheum. 2008:58(5):1505-15.

11. Lequerre T, Quartier P, Rosellini D, Alaoui F, De Bandt M, Mejjad O. Interleukin-1 receptor antagonist (anakinra) treatment in patients with systemic-onset juvenile idiopathic arthritis or adult onset Still disease: preliminary experience in France. Ann Rheum Dis. 2008;67(3):302-8.

12. Pascual V, Allantaz F, Arce E, Punaro M, Banchereau J. Role of interleukin-1 (IL-1) in the pathogenesis of systemic onset juvenile idiopathic arthritis and clinical response to IL-1 blockade. J Exp Med. 2005;201(9):1479-86.

13. Quartier P, Allantaz F, Cimaz R, Pillet P, Messiaen C, Bardin C, et al. A multicentre, randomised, double-blind, placebo-controlled trial with the interleukin-1 receptor antagonist anakinra in patients with systemic-onset juvenile idiopathic arthritis (ANAJIS trial). Ann Rheum Dis. 2011;70(5):747-54.

14. Woo P, Wilkinson N, Prieur AM, Southwood T, Leone V, Livermore P, et al. Open label phase II trial of single, ascending doses of MRA in Caucasian children with severe systemic juvenile idiopathic arthritis: proof of principle of the efficacy of 
IL-6 receptor blockade in this type of arthritis and demonstration of prolonged clinical improvement. Arthritis Res Ther. 2005;7(6):R1281-8.

15. Woo P. Anakinra treatment for systemic juvenile idiopathic arthritis and adult onset Still disease. Ann Rheum Dis. 2008;67(3):281-2.

16. Yokota S, Tanaka T, Kishimoto T. Efficacy, safety and tolerability of tocilizumab in patients with systemic juvenile idiopathic arthritis. Ther Adv Musculoskelet Dis. 2012;4(6):387-97.

17. Yokota S, Imagawa T, Mori M, Miyamae T, Takei S, Iwata N, et al. Long-term treatment of systemic juvenile idiopathic arthritis with tocilizumab: results of an open-label extension study in Japan. Ann Rheum Dis. 2013;72(4):627-8,

18. Ishikawa S, Mima T, Aoki C, Yoshio-Hoshino N, Adachi Y, Imagawa T, et al. Abnormal expression of the genes involved in cytokine networks and mitochondrial function in systemic juvenile idiopathic arthritis identified by DNA microarray analysis. Ann Rheum Dis. 2009;68(2):264-72.

19. Fall N, Barnes M, Thornton S, Luyrink L, Olson J, llowite NT, et al. Gene expression profiling of peripheral blood from patients with untreated newonset systemic juvenile idiopathic arthritis reveals molecular heterogeneity that may predict macrophage activation syndrome. Arthritis Rheum. 1956; 2007:3793-804.

20. Barnes MG, Grom AA, Thompson SD, Griffin TA, Pavlidis $P$, Itert $L$, et al. Subtype-specific peripheral blood gene expression profiles in recent-onset juvenile idiopathic arthritis. Arthritis Rheum. 1960;2009:2102-12.

21. Jarvis JN, Petty HR, Tang Y, Frank MB, Tessier PA, Dozmorov I, et al. Evidence for chronic, peripheral activation of neutrophils in polyarticular juvenile rheumatoid arthritis. Arthritis Res Ther. 2006;8(5):R154.

22. Jarvis JN, Jiang K, Petty HR, Centola M. Neutrophils: the forgotten cell in JIA disease pathogenesis. Pediatr Rheumatol Online J. 2007;5:13.

23. Jarvis JN, Jiang K, Frank MB, Knowlton N, Aggarwal A, Wallace CA, et al. Gene expression profiling in neutrophils from children with polyarticular juvenile idiopathic arthritis. Arthritis Rheum. 2009;60(5):1488-95.

24. Amulic B, Cazalet C, Hayes GL, Metzler KD, Zychlinsky A. Neutrophil function: from mechanisms to disease. Annu Rev Immunol. 2012;30:459-89.

25. Kolivras A, Theunis A, Ferster A, Lipsker D, Sass U, Dussart A, et al. Cryopyrinassociated periodic syndrome: an autoinflammatory disease manifested as neutrophilic urticarial dermatosis with additional perieccrine involvement. J Cutan Pathol. 2011;38(2):202-8.

26. Manukyan G, Petrek M, Kriegova E, Ghazaryan K, Fillerova R, Boyajyan A. Activated phenotype of circulating neutrophils in familial Mediterranean fever. Immunobiology. 2013;218(6):892-8.

27. Petty RE, Southwood TR, Manners P, Baum J, Glass DN, Goldenberg J, et al. International League of Associations for Rheumatology classification of juvenile idiopathic arthritis: second revision, Edmonton, 2001. J Rheumatol. 2004;31(2):390-2.

28. Giannini EH, Ruperto N, Ravelli A, Lovell DJ, Felson DT, Martini A. Preliminary definition of improvement in juvenile arthritis. Arthritis Rheum. 1997:40(7):1202-9.

29. Omoyinmi E, Hamaoui R, Pesenacker A, Nistala K, Moncrieffe H, Ursu S, et al. Th1 and Th17 cell subpopulations are enriched in the peripheral blood of patients with systemic juvenile idiopathic arthritis. Rheumatology (Oxford). 2012;51(10):1881-6.

30. Wu Z, Irizarry RA. Stochastic models inspired by hybridization theory for short oligonucleotide arrays. J Comput Biol. 2005;12(6):882-93.

31. Ding LH, Xie Y, Park S, Xiao G, Story MD. Enhanced identification and biological validation of differential gene expression via lllumina wholegenome expression arrays through the use of the model-based background correction methodology. Nucleic Acids Res. 2008;36(10):e58.

32. Subramanian A, Tamayo P, Mootha VK, Mukherjee S, Ebert BL, Gillette MA, et al. Gene set enrichment analysis: a knowledge-based approach for interpreting genome-wide expression profiles. Proc Natl Acad Sci U S A. 2005;102(43):15545-50

33. Foell D, Frosch M, Sorg C, Roth J. Phagocyte-specific calcium-binding S100 proteins as clinical laboratory markers of inflammation. Clin Chim Acta. 2004;344(1-2):37-51

34. Frosch M, Ahlmann M, Vogl T, Wittkowski H, Wulffraat N, Foell D, et al. The myeloid-related proteins 8 and 14 complex, a novel ligand of toll-like receptor 4 , and interleukin-1 beta form a positive feedback mechanism in systemiconset juvenile idiopathic arthritis. Arthritis Rheum. 2009;60(3):883-91.

35. Wittkowski H, Frosch M, Wulffraat N, Goldbach-Mansky R, Kallinich $T$ Kuemmerle-Deschner J, et al. S100A12 is a novel molecular marker differentiating systemic-onset juvenile idiopathic arthritis from other causes of fever of unknown origin. Arthritis Rheum. 2008;58(12):3924-31.
36. Ji C, Chen X, Gao C, Jiao L, Wang J, Xu G, et al. IL-6 induces lipolysis and mitochondrial dysfunction, but does not affect insulin-mediated glucose transport in 3T3-L1 adipocytes. J Bioenerg Biomembr. 2011;43(4):367-75.

37. Grayson PC, Kaplan MJ. At the Bench: Neutrophil extracellular traps (NETs) highlight novel aspects of innate immune system involvement in autoimmune diseases. J Leukoc Biol. 2015.

38. Lee HM, Sugino H, Aoki C, Nishimoto N. Underexpression of mitochondrialDNA encoded ATP synthesis-related genes and DNA repair genes in systemic lupus erythematosus. Arthritis Res Ther. 2011;13(2):R63.

39. Zhou R, Yazdi AS, Menu P, Tschopp J. A role for mitochondria in NLRP3 inflammasome activation. Nature. 2011;469(7329):221-5.

40. van de Veerdonk FL, Smeekens SP, Joosten LA, Kullberg BJ, Dinarello CA, van der Meer JW, et al. Reactive oxygen species-independent activation of the IL-1 beta inflammasome in cells from patients with chronic granulomatous disease. Proc Natl Acad Sci U S A. 2010;107(7):3030-3.

41. Cruz CM, Rinna A, Forman HJ, Ventura AL, Persechini PM, Ojcius DM. ATP activates a reactive oxygen species-dependent oxidative stress response and secretion of proinflammatory cytokines in macrophages. J Biol Chem. 2007;282(5):2871-9.

42. Bulua AC, Simon A, Maddipati R, Pelletier M, Park H, Kim KY, et al. Mitochondrial reactive oxygen species promote production of proinflammatory cytokines and are elevated in TNFR1-associated periodic syndrome (TRAPS). J Exp Med. 2011;208(3):519-33.

43. Fuchs $T$, Puellmann $\mathrm{K}$, Scharfenstein $\mathrm{O}$, Eichner $\mathrm{R}$, Stobe $\mathrm{E}$, Becker $\mathrm{A}$, et al. The neutrophil recombinatorial TCR-like immune receptor is expressed across the entire human life span but repertoire diversity declines in old age. Biochem Biophys Res Commun. 2012;419(2):309-15.

44. Puellmann K, Kaminski WE, Vogel M, Nebe CT, Schroeder J, Wolf H, et al. A variable immunoreceptor in a subpopulation of human neutrophils. Proc Natl Acad Sci U S A. 2006;103(39):14441-6.

45. Jarvis JN, Dozmorov I, Jiang K, Frank MB, Szodoray P, Alex P, et al. Novel approaches to gene expression analysis of active polyarticular juvenile rheumatoid arthritis. Arthritis Res Ther. 2004;6(1):R15-32.

46. Jiang K, Frank M, Chen Y, Osban J, Jarvis JN. Genomic characterization of remission in juvenile idiopathic arthritis. Arthritis Res Ther. 2013;15(4):R100.

47. De Benedetti F, Brunner HI, Ruperto N, Kenwright A, Wright S, Calvo I, et al. Randomized trial of tocilizumab in systemic juvenile idiopathic arthritis. N Engl J Med. 2012;367(25):2385-95.

\section{Submit your next manuscript to BioMed Central and we will help you at every step:}

- We accept pre-submission inquiries

- Our selector tool helps you to find the most relevant journal

- We provide round the clock customer support

- Convenient online submission

- Thorough peer review

- Inclusion in PubMed and all major indexing services

- Maximum visibility for your research

Submit your manuscript at www.biomedcentral.com/submit
Biomed Central 